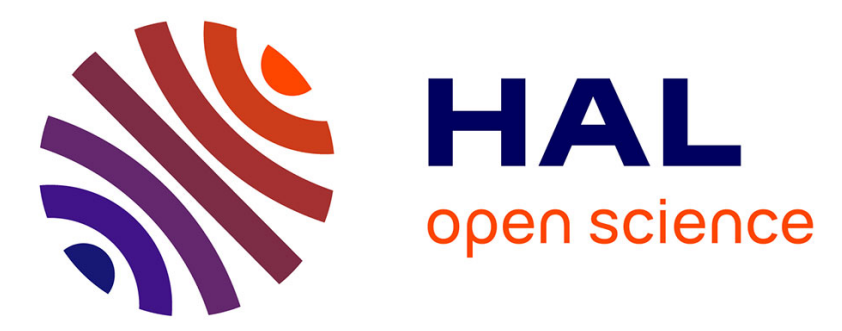

\title{
Prise en compte de l'anisotropie des collisions ion-atome sur le transport des ions par simulation de Monte-Carlo
}

A. Hennad, Olivier Eichwald, Mohammed Yousfi, O. Lamrous

\section{To cite this version:}

A. Hennad, Olivier Eichwald, Mohammed Yousfi, O. Lamrous. Prise en compte de l'anisotropie des collisions ion-atome sur le transport des ions par simulation de Monte-Carlo. Journal de Physique III, 1997, 7 (9), pp.1877-1892. 10.1051/jp3:1997229 . jpa-00249687

\section{HAL Id: jpa-00249687 https://hal.science/jpa-00249687}

Submitted on 1 Jan 1997

HAL is a multi-disciplinary open access archive for the deposit and dissemination of scientific research documents, whether they are published or not. The documents may come from teaching and research institutions in France or abroad, or from public or private research centers.
L'archive ouverte pluridisciplinaire HAL, est destinée au dépôt et à la diffusion de documents scientifiques de niveau recherche, publiés ou non, émanant des établissements d'enseignement et de recherche français ou étrangers, des laboratoires publics ou privés. 


\title{
Prise en compte de l'anisotropie des collisions ion-atome sur le transport des ions par simulation de Monte-Carlo
}

\author{
A. Hennad, O. Eichwald, M. Yousfi (*) et O. Lamrous \\ Université Paul Sabatıer, CPAT $\left({ }^{* *}\right), 118$ Route de Narbonne, 31062 Toulouse Cedex 4, France
}

(Reçu le 10 janvier 1997, révisé le 15 mai 1997, accepté le 27 mal 1997)

PACS.52 20.Hv - Atomic, molecular, ion and heavy-particle collisions

PACS.51.50.+v - Electrical properties (ionization, breakdown, electron and ion mobility, etc.)

PACS 52.25.Fi - Transport properties

Résumé. - Cet article est consacré à la détermination des sections efficaces différentielles et intégrales ion-atome nécessaires au calcul des coefficients de transport des ions dans les gaz faiblement ionisés. Dans le cas du système $\mathrm{Ar}^{+} / \mathrm{Ar}$ et pour des intervalles d'énergie allant jusqu'à quelques dizaines d'eV, les sections efficaces sont obtenues à partir des potentiels d'interaction de polarisation pour les faibles énergies et de Lennard-Jones pour les énergies plus élevées. La méthode de calcul des sections efficaces basée sur la mécanıque classique a d'abord été validée par comparaıson des sections efficaces différentielles mesurées et calculées Ensuite, ces sections efficaces ont été utilisées dans un code de simulatıon statistique de Monte-Carlo du transport des ions $\mathrm{Ar}^{+}$dans l'Argon à la température ambiante $(300 \mathrm{~K})$. Les coefficients de transport (vitesse de dérive, mobilité ionique et coefficient de diffusion) obtenus sont en bon accord avec les mesures de tube de dérive de la littérature confirmant ansi la validation de la méthode de détermination des sections efficaces.

\begin{abstract}
This paper is devoted to the determination of the differential and integral collision cross sections needed for the calculation of the transport coefficient of ions in weakly ionized gases In the case of $\mathrm{Ar}^{+} / \mathrm{Ar}$ system and for energy interval varying up to $100 \mathrm{eV}$, the cross sections are obtained from the interaction potential of polarization for low energies and of Lennard-Jones for higher energies The calculation method of the collision cross sections based on the classical mechanics has been first validated from comparisons of measured and calculated differential cross sections. Then, these cross sections have been used in a Monte-Carlo code for simulation of the transport of $\mathrm{Ar}^{+}$ions in Ar gas at room temperature (300 K). The obtained transport coefficients (1on mobility, drift velocity and diffusion coefficient) are in good agreement with the drift tube measurements given in the literature thus confirming the valıdity of the method of collision cross section calculation
\end{abstract}

\section{Introduction}

La modélisation des décharges en général, et l'étude du transport des ions dans les plasmas froids non-thermiques en particulier, nécessitent notamment la connaissance des sections efficaces de collision ion-atome. Dans les milieux gazeux formés par les décharges hors équilibre,

$\left({ }^{*}\right)$ Auteur auquel doit être adressée la correspondance (e-mail : yousfi@cpa29.ups-tlse.fr)

$\left({ }^{* *}\right)$ ESA du CNRS n ${ }^{\circ} 5002$ 
en raison des ordres de grandeur des énergies ioniques mises en jeu (quelques électron-volt au maximum), ce sont surtout les collisions élastiques et de transfert de charge résonnant ou non-résonnant qui dominent, les autres processus inélastiques (excitation. ionisation, etc.) sont moins probables. Dans tous les cas, l'étude de l'ensemble de ces processus est essentielle car c'est en partie à travers eux que se font les transferts d'impulsion et d'énergie (comme par exemple les vents électriques dans les décharges couronnes). Ces processus peuvent être également déterminant dans la composition du plasma (création de nouvelles particules par transfert de charge, dissociation, ionisation, etc.). C'est pourquoi dans cet article, on s'intéressa principalement aux processus ion-atome élastiques et aussi de transfert de charge dont le rôle est loin d'être négligeable dans le transport des ions dans les gaz atomiques car ils contribuent à la diffusion, la viscosité, la conductivité électrique et thermique, etc.

De plus, le transport des ions est sensible non seulement à l'amplitude de la section efficace mais également à la déviation après la collision qui qualifie le degré d'anisotropie de la collision. C'est la raison pour laquelle on s'intéresse aux méthodes de détermination des sections efficaces intégrales et surtout différentielles, afin de les utiliser par la suite dans le code de Monte-Carlo pour calculer les paramètres de transport des ions dans les gaz atomiques en tenant compte de l'anisotropie des collisions. Les sections efficaces différentielles sont obtenues à partir des potentiels d'interaction dont le choix est déterminant sur le transport des ions. Les potentiels d'interactions choisis sont le potentiel de polarisation pour les faibles énergies ioniques (inférieures à environ $0,1 \mathrm{eV}$ dans le cas du système $\mathrm{Ar}^{+} / \mathrm{Ar}$ étudié dans ce travail) et le potentiel Lennard-Jones pour les énergies plus élevées. Ces choix de potentiels nous permettent ensuite, connaissant la trajectoire de la particule incidente largement décrite dans la littérature [1-3], de déterminer pour chaque énergie relative l'angle de déviation à partir de la relation entre le potentiel et le paramètre d'impact. Les sections efficaces différentielles sont ensuite obtenues à partir de l'angle de déviation en utilisant comme approximation les expressions basées sur la mécanique classique. Une solution numérıque appropriée sera développée pour traiter le problème de la singularité qui apparaît dans le calcul de la section efficace différentielle issues du potentiel Lennard-Jones. Dans le but de mieux comprendre l'influence du choix de l'énergie du puits et du diamètre de la collisıon dans le cas du potentiel Lennard-Jones, une étude paramétrique sera effectuée en analysant l'angle de déviation et les sections efficaces différentielles et intégrales. Ensuite, la validation de l'utilisation de l'approche classique sera discutée en comparant les sections efficaces calculées et mesurées dans le cas du système $\mathrm{Ar}^{+} / \mathrm{Ar}$. La confirmation de cette validation du calcul des sections efficaces a ensuite été discutée en déterminant les coefficient de transport (vitesse de dérive, mobilité, coefficients de diffusion, etc.) des ions $\mathrm{Ar}^{+}$dans Ar par simulation statistique de Monte-Carlo qui prend en compte l'anisotropie de la collision à partir des sections efficaces différentielles.

\section{Méthode de calcul}

\subsection{MÉthodes de CAlCul de L'angle de déviation et DEs SeCtions efFicaces. -} Étant donnée la complexité de la structure des atomes, la connaissance du potentiel d'interaction réel est difficilement envisageable. En revanche, on peut utiliser des modèles mathématiques qui simulent d'une manière raisonnable le potentıel d'interaction ion-atome. Le choix du potentiel d'interaction ion-atome peut se faire à partir de deux critères. Il faut considérer d'une part les difficultés de calcul associées à la forme du potentiel d'interaction et d'autre part le degré de précision désiré dans la description des interactions ion-atome. Il est clair que ces deux points de vue peuvent être antagonistes car les fonctions potentielles voisines de la réalité physique conduisent souvent à des calculs laborieux pouvant entraîner des imprécisions d'ordre numérique. La question qui se pose est de savoir quelles sont les formes du potentiel 
que l'on peut considérer avec une bonne approximation pour les collisions ion-atome qui nous intéressent. Le choix s'est orienté vers le potentiel de polarisation pour des régions d'énergies relativement faibles et le potentiel de Lennard-Jones pour les valeurs d'énergie plus importantes. Les domaines d'énergies choisis ne sont pas suffisamment élevés pour exiger l'utilisation d'un troisième type de potentiel pour les plus hautes énergies. Il faut cependant savoir que, de manière générale, l'interaction ion-atome peut être divisée en longue, moyenne et courte portée. On peut se référer aux ouvrages qui traitent en détails ces différents types d'interaction (Hirschfelder et al. [1] et [4], Certain et Bruch [5], Maitland et al. [6], Jancel et Kahan [7], et Gray et Gubbins [8]).

Le potentiel de polarisation est choisi dans ce travail parce qu'il est généralement bien adapté pour les interactions ion-atome de faible énergie et de longue portée. Il s'écrit :

$$
V(r)=-\frac{\alpha e^{2}}{2 r^{4}}
$$

où $e$ est la charge électrique de l'ion projectile, et $\alpha$ le coefficient de polarisation de l'atome cible. Ce potentiel de polarisation ou d'induction prend naissance principalement à partir de l'interaction de la charge ionique avec le moment multipolaire induit de l'atome Dans ce cas, les ions peuvent avoir deux types de trajectoires ; celles de forme hyperbolique et celles en spirale correspondant respectivement à des grands et faibles moments angulaires. Dans le cas des trajectoires spirales, l'ion piégé dans son orbite peut effectuer plusieurs rotations jusqu'à son éjection par une force répulsive (voir par exemple McDaniel [9]). Un tel phénomène peut altérer de façon substantielle l'ordre de grandeur des paramètres de transport des ions. Mais compte tenu des limites inférieures des énergies ioniques moyennes mises en jeu dans les décharges électriques qui nous intéressent (supérieures à l'énergie du gaz), le phénomène de piégeage peut être négligé.

Le potentiel de type Lennard-Jones est davantage utilisé pour les interáctions neutre-neutre mais il représente aussi une bonne approximation pour les interactions ion-atome surtout dans les régions d'énergie intermédiaire $[10,11]$. Dans notre cas, il s'écrit :

$$
V(r)=4 \varepsilon_{\mathrm{p}}\left[\left(\frac{d}{r}\right)^{12}-\left(\frac{d}{r}\right)^{6}\right]
$$

Le diamètre de la collision $d$ correspond à la valeur du potentiel pour laquelle $V(d)=0 . \varepsilon_{\mathrm{p}}$ représente la profondeur maximale du puits. Le terme attractif en $(d / r)^{6}$ représente correctement les interactions dipôle induit-dipôle induit entre atomes (interaction longue portée). Le terme $(d / r)^{12}$ du potentiel de Lennard-Jones correspond à la branche répulsive représentant les interactions de courte portée. Dans cet article, nous avons choisi pour ce terme la puissance 12. Désormais, quand nous faisons référence au potentiel de Lennard-Jones nous l'entendons avec la forme (2). La présence des deux termes (attractif et répulsif) est justifiée par le fait que lorsque l'ion s'approche d'un atome, les forces interatomiques sont d'abord attractives, puis quand les nuages électroniques commencent à s'interpénétrer les forces d'attraction diminuent régulièrement et à plus faible distance, deviennent répulsives.

Le calcul de la section efficace de diffusion d'un faisceau d'ions en mécanique quantique, nécessite habituellement la considération d'un nombre extrêmement large d'ondes partielles nécessitant ainsi des temps de calcul prohibitif. Heureusement, les grands angles de déviation peuvent être souvent traités à l'aide de la mécanique classique plus simple d'emploi, et il peut devenir nécessaire de prendre en compte la nature quantique du mouvement des particules qui entrent en collision seulement aux faibles angles de déviation. Cependant, plusieurs méthodes de calcul basées sur la méthode classique ont été développées et appliquées au calcul de la 


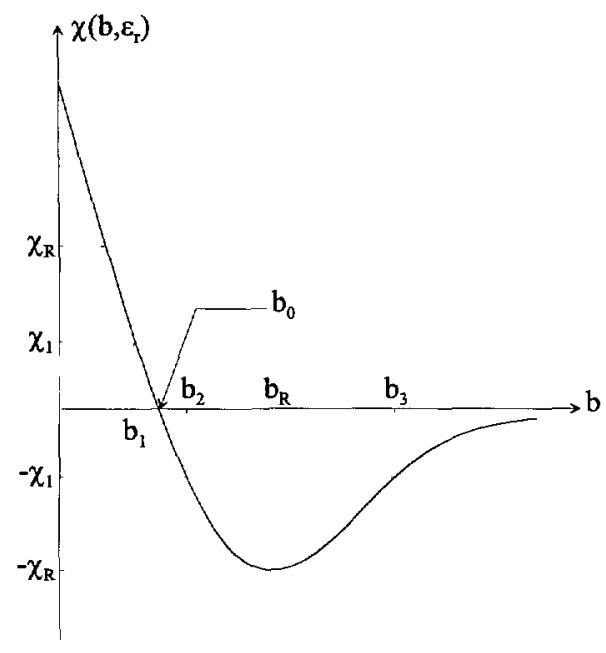

Fig. 1. - Angle de déviation incluant la définition des différents paramètres d'impacts $b_{0}, b_{1}, b_{2}, b_{3}$ et $b_{R}$ dans le cas d'un potentiel de type Lennard-Jones.

[Deviation angle involving the definitıons of the various impact parameters $b_{0}, b_{1}, b_{2}, b_{3}$ and $b_{R}$ in the case of the Lennard-Jones potential.]

diffusion à faible angle de déviation $[12,13]$. Dans ce qui suit, on va décrire la méthode utilisée, basée sur la mécanique classique, de la diffusion élastique dans le cas du potentiel LennardJones qui est un potentiel à symétrie sphérique.

Le problème de collision de deux particules est réduit au mouvement d'une seule particule sous l'influence d'un potentiel central dans le repère de centre de masse. La fonction de déviation $\chi$ de l'interaction ion-atome dépendant du paramètre d'impact $b$ et de l'énergie relative $\varepsilon_{\mathrm{r}}$, s'obtient directement à partir de la conservation d'énergie et du moment angulaire $[14,15]$ :

$$
\chi\left(b, \varepsilon_{\mathrm{r}}\right)=\pi-2 \int_{0}^{\rho_{0}} \frac{\mathrm{d} \rho}{\left(1-\rho^{2}-V(\rho) / \varepsilon_{\mathrm{r}}\right)^{1 / 2}} .
$$

Dans cette expression, nous avons effectué un changement de variable $\rho=b / r ; \rho_{0}$ étant la valeur la plus petite de deux racines réelles solution du polynôme au dénominateur $\left(1-\rho^{2}-\right.$ $\left.V(\rho) / \varepsilon_{\mathrm{r}}=0\right)$. Le comportement typique de la fonction de déviation $\chi\left(b, \varepsilon_{\mathrm{r}}\right)$ pour un potentiel de Lennard-Jones est représenté sur la figure 1. Les interactions avec un angle de déviation positif sont attribuées à la partie répulsive du potentiel Lennard-Jones (interaction de courte portée) ayant des faibles valeurs du paramètre d'impact. L'angle de déviation décroît quand le paramètre d'impact augmente, jusqu'à ce que les forces attractives et répulsives se neutralisent mutuellement pour $b=b_{0}$, ce qui donne une déviation nulle. Pour de grandes valeurs de $b$, la partie attractive du potentiel Lennard-Jones (interaction longue portée) l'emporte et conduit au changement de direction de la fonction de déviation qui converge vers zéro quand $b$ tend vers l'infini. D'autre part, lorsque $b=b_{R}$, on observe que la fonction de déviation présente un extremum correspondant à l'angle $\chi_{R}$ (voir Fig. 1). Etant donné qu'un angle de déviation peut correspondre à plusieurs paramètres d'impacts (voir Fig. 1 où $\left|\chi_{1}\right|$ correspond à $b_{1}, b_{2}$ et $b_{3}$ ), la détermination de la section efficace différentielle $\sigma\left(\chi, \varepsilon_{\mathrm{r}}\right)$ nécessite l'addition de tous les 
paramètres d'impact qui conduisent à la même valeur de l'angle de déviation :

$$
\sigma\left(\chi, \varepsilon_{\mathrm{r}}\right)=\left|\frac{1}{\sin \chi} \sum_{\imath} b_{\imath} \frac{\mathrm{d} b_{\imath}}{\mathrm{d} \chi}\right| .
$$

De manière générale, pour les angles de déviation $\chi<\chi_{R}$, il y a trois paramètres d'impact $b_{1}, b_{2}$ et $b_{3}$ qui interviennent dans le calcul de la section efficace différentielle, tandis que pour $\chi>\chi_{R}$, un seul paramètre d'impact $b_{1}$ intervient dans le calcul de la section efficace différentielle. Cette méthode de détermination de la section efficace différentielle a été développée par McDowell et Coleman [15], ou bien par Pauly et Toennies [16], ou encore par Bransden et Joachain [17].

Il est important de noter la présence d'une singularité dans l'expression classique de la section efficace différentielle (4) incluant le terme $(\mathrm{d} \chi / \mathrm{d} b)^{-1}$ qui s'annule pour $\chi=\chi_{R}$. L'angle $\chi_{R}$ est l'angle de déviation minimum appelé aussi l'angle de Rainbow par analogie aux phénomènes optiques de diffusion de la lumière sur les fines gouttelettes de pluie donnant naissance à l'arcen-ciel. La déviation des ions atomiques par d'autres atomes est similaire à la déviation de la lumière dans une goutte d'eau (phénomène de l'arc-en-ciel) Ce phénomène a été souligné pour la première fois par Mason [18].

Afin de résoudre le problème de singularité qui se pose pour la détermination de la section efficace différentielle pour $\chi_{R}$, la fonction de déviation peut être développée au voisinage de l'angle $\chi_{R}$ comme suit :

$$
\chi\left(b . \varepsilon_{\mathrm{r}}\right)=\chi_{R}+\tilde{p}\left(b-b_{R}\right)^{2}
$$

avec

$$
\tilde{p}=\frac{1}{2}\left(\frac{\mathrm{d}^{2} \chi}{\mathrm{d} b^{2}}\right)_{\chi=\chi_{R}} .
$$

À partir de ce développement, le comportement de la partie "Rainbow" de la section efficace différentielle (c'est-à-dire au voisinage de l'angle $\chi_{R}$ ) est facilement dérivable en considérant seulement les contributions $b_{2}\left|\mathrm{~d} b_{2} / \mathrm{d} \chi\right|$ et $b_{3}\left|\mathrm{~d} b_{3} / \mathrm{d} \chi\right|$ dans l'équation (4) et en négligeant la contribution supplémentaire $b_{1}\left|\mathrm{~d} b_{1} / \mathrm{d} \chi\right|$ (voir Fig. 1). Si du côté sombre ("dark side") de l'angle Rainbow $\left(\chi>\chi_{R}\right)$ seul le terme en $b_{1}$ de la relation (4) intervient dans le calcul de $\sigma\left(\chi, \varepsilon_{\mathrm{r}}\right)$, du côte clair ("bright side") $\left(\chi<\chi_{R}\right)$, la section efficace différentielle de Rainbow se calcule à partir de :

$$
\sigma\left(\chi, \varepsilon_{\mathrm{r}}\right)=\frac{b_{R}}{\sin \chi_{R}\left|\tilde{p}\left(\chi-\chi_{R}\right)\right|^{1 / 2}} .
$$

Dans l'étude des propriétés d'un système ion-atome, où l'on désire évaluer l'importance des collisions élastiques, on a souvent besoin de définir leurs sections efficaces différentielles comme on l'a fait dans ce paragraphe, ainsi que sa section efficace de transfert de la quantité de mouvement $Q_{m}\left(\varepsilon_{\mathrm{r}}\right)$ :

$$
Q_{m}\left(\varepsilon_{\mathrm{r}}\right)=2 \pi \int_{0}^{\pi} \sigma\left(\chi, \varepsilon_{\mathrm{r}}\right)(1-\cos \chi) \sin \chi \mathrm{d} \chi=2 \pi \int_{0}^{\infty}(1-\cos \chi) b \mathrm{~d} b
$$

2.2. MÉthode de monte-Carlo. - Une fois connues les sections efficaces différentielles et intégrales, il est possible de prendre en compte l'anisotropie des collisions pour le calcul des coefficients de transport des ions dans les gaz faiblement ionisés en effectuant la simulation du transport des ions par une méthode de Monte-Carlo qui est décrite en détail dans la littérature $[19,20]$. Il faut tout de même noter que c'est une méthode dite au coup par coup où les ions germes sont traités un par un depuis leur création jusqu'à leur disparition soit par certains processus collisionnels (transfert de charge asymétrique) ou par dépassement des limites du domaine de simulation. Dans un cas comme dans l'autre, tout ion émis à partir d'une 
position $r_{0}$, avec une vitesse $v_{0}$ à l'instant $t_{0}$ effectue une succession de vols libres ponctués par des collisions élastiques ou inélastiques supposées locales et instantanées avant d'atteindre les limites spatio-temporelles du domaine. Durant chaque temps de vol libre, la position et la vitesse de l'ion avant le choc sont déterminées par les lois de la mécanique classique, tandis que la nature de la collision et la vitesse de l'ion juste après le choc dépendent des sections efficaces différentielles et intégrales. Pendant les collisions successives, certaines informations (positions, vitesses, etc.) sont stockées dans le but de calculer, à partir de méthodes d'échantillonnage appropriées, les fonctions de distribution et les paramètres de transport des ions. La simulation est arrêtée lorsque tous les ions sont traités. Il faut souligner que l'algorithme de Monte-Carlo est optimisée en déterminant le temps entre deux collisions successives $t_{\mathrm{vol}}$ à l'aide de la méthode classique des collisions nulles $[19,20]$ en utilisant la relation suivante :

$$
t_{\mathrm{vol}}=-\frac{\ln \left(R_{\mathrm{vol}}\right)}{\nu_{\mathrm{tot}}}
$$

où $\nu_{\text {tot }}$ est la fréquence totale de collision ion-atome incluant la fréquence totale des collisions réelles $\nu_{1}$ et une fréquence de collision dite nulle $\nu_{\text {nul }}$ choisie de telle sorte que la fréquence $\nu_{\text {tot }}$ soit toujours constante $\left(\nu_{\text {tot }}=\nu_{1}+\nu_{\text {nul }}=\right.$ constante). Dans ce cas, le temps de vol (au lieu d'être déterminé à l'aide d'une relation intégrale pénalisante d'un point de vue temps de calcul) est obtenu à l'aide de la relation (8) par simple tirage d'un nombre aléatoire $R_{\text {vol }}$ uniformément distribué dans l'intervalle $\{0,1\}$. Ceci permet un gain de temps de calcul d'environ un facteur 5. Lors du traitement des collisions par la méthode de Monte-Carlo, la prise en compte de l'anisotropie de la collision s'effectue en calculant l'angle de déviation $\chi_{\mathrm{p}}$ de l'ion diffusé après le choc à l'aide de la relation (9) par simple tirage de nombres aléatoires $R_{\chi_{\mathrm{p}}}$ uniformément distribués dans l'intervalle $\{0,1\}$ :

$$
R_{\chi_{\mathrm{p}}}=\frac{\int_{0}^{\chi_{\mathrm{p}}} \sigma\left(\chi, \varepsilon_{\mathrm{r}}\right) \sin \chi \mathrm{d} \chi}{\int_{0}^{\pi} \sigma\left(\chi, \varepsilon_{\mathrm{r}}\right) \sin \chi \mathrm{d} \chi} .
$$

\section{Résultats}

3.1. Étude paramétrique. - Dans cette partie, on discute l'influence du diamètre de la collision $d$, et la profondeur de l'énergie du puits du potentiel $\varepsilon_{\mathrm{p}}$ sur l'angle de déviation, les sections efficaces différentielle et intégrale. Dans la littérature les paramètres $d$ et $\varepsilon_{\mathrm{p}}$ pour les interactions ion-atome sont quasi inexistants ; c'est pourquoi nous montrons par la suite, comment obtenir ces paramètres à partir de l'observation de leur influence sur les sections efficaces et également par ajustement de ces paramètres en comparant les sections efficaces mesurées et calculées $[19,21]$.

La figure 2 montre la variation de l'angle de déviation $\chi(b)$ pour plusieurs valeurs du diamètre de la collision d. On observe que l'augmentation de $d$ entraîne un décalage de la fonction de déviation vers des valeurs plus élevées de $b$ sans changer l'ordre de grandeur des angles de déviation et en particulier l'angle de Rainbow. Ce décalage est tout simplement dû au fait que l'augmentation du diamètre n'entraîne qu'une simple translation du potentiel sans modifier l'amplitude des forces d'attraction et de répulsion. Ceci peut être aisément observée sur la figure 3 qui montre une représentation schématique de la déviation de la particule $M_{\mathrm{r}}$ dans le repère du centre de masse pour deux diamètres de la collision différents. En revanche, la variation de la profondeur du puits de potentiel $\varepsilon_{\mathrm{p}}$ fait varier l'amplitude de l'angle Rainbow (voir Fig. 4). Ceci peut s'expliquer en se basant sur l'approximation d'impulsion donnée par Johnson [22] pour le potentiel de Lennard-Jones qui montre que dans le cas d'un ion incident rapide l'angle Rainbow est directement proportionnel à l'énergie du puits. 


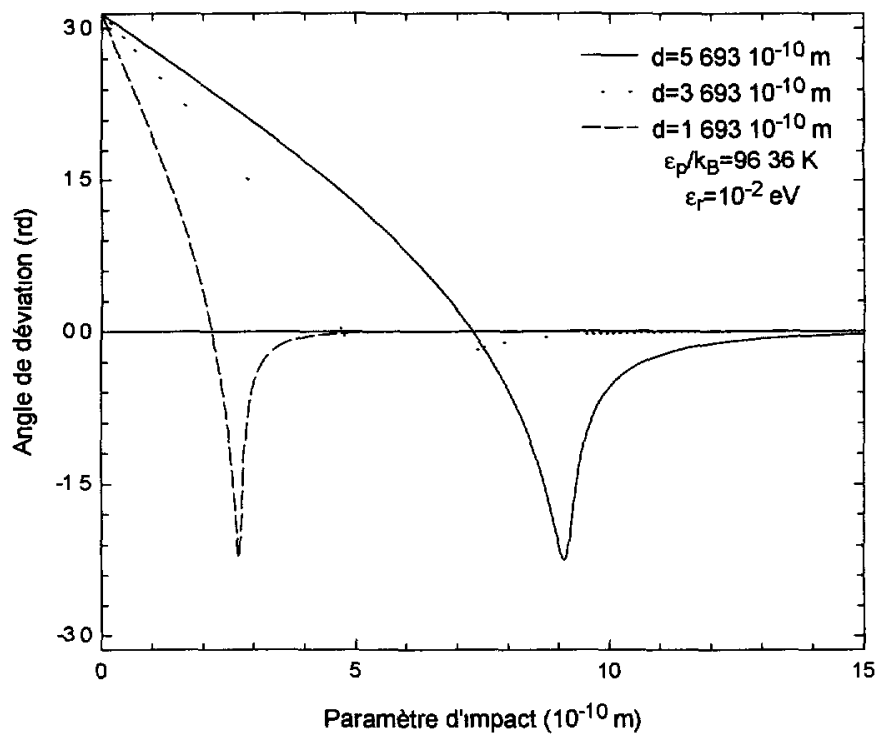

Fig. 2. - Angle de déviation en fonction du paramètre d'impact pour différentes valeurs du diamètre de la collision $d$ dans le cas d'un potentiel de type Lennard-Jones.

[Deviation angle as a function of the impact parameter for different values of the collision diameter $d$ in the case of the Lennard-Jones $[6,12]$ potential ]

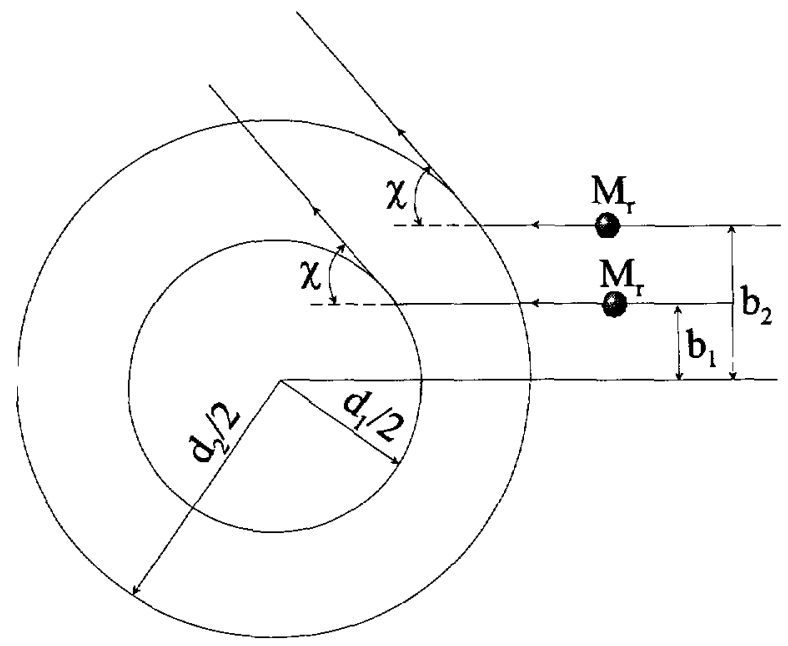

Fig. 3. - Représentation schématıque de la dévıation d'une particule $M_{\mathrm{r}}$ dans le repère du centre de masse pour deux valeurs du diamètre de la collision dans le cas d'un potentiel de type Lennard-Jones.

[Schematic representation of the deviation of a particle $M_{\mathrm{r}}$ in the Center of Mass framework for two values of the collision diameter $d$ in the case of the Lennard-Jones potential.] 


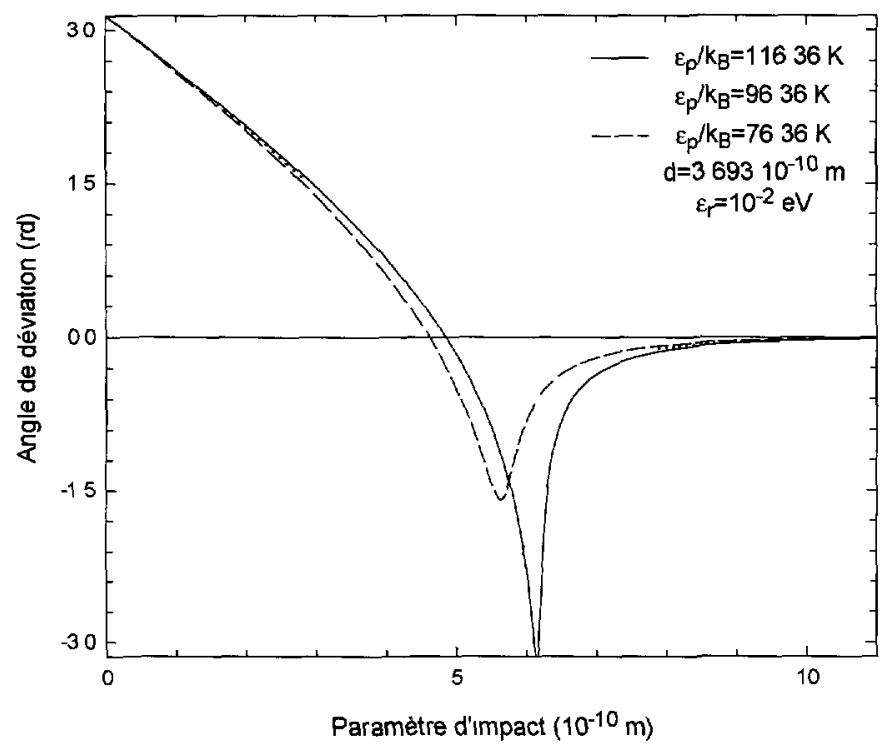

Fig. 4 - Angle de déviation en fonction du paramètre d'impact pour différentes valeurs de l'énergie du puits $\varepsilon_{\mathrm{p}}$ dans le cas d'un potentiel de type Lennard-Jones.

[Deviation angle as a function of the impact parameter for different values of the energy of the potential well depth $\varepsilon_{\mathrm{p}}$ in the case of the Lennard-Jones potential.]

Les figures $5 \mathrm{a}$ et $5 \mathrm{~b}$ représentent la section efficace différentielle pour respectivement plusieurs diamètres de la collision et énergies du puits. On observe comme prévu que la section efficace diminue en même temps que le diamètre de la collision car la surface d'interaction diminue elle aussi Cependant la variation de la profondeur du puits affecte beaucoup moins la section efficace différentielle sauf au point de la singularité $\left(\mathrm{d} \chi /\left.\mathrm{d} b\right|_{\chi_{=\chi_{R}}}=0\right)$ En effet, sur la figure 5b on observe que lorsque l'énergie du puits augmente, le point de discontinuité se déplace (conformément au comportement déjà décrit dans le cas de la figure 4) vers les valeurs de l'angle de déviation les plus élevées. La discontinuité n'apparaît pas sur la courbe correspondant à l'énergie la plus grande $\left(\varepsilon_{\mathrm{p}} / k_{\mathrm{B}}=116,36 \mathrm{~K}\right)$ tout simplement parce que le point de discontinuité se trouve au delà de $\chi=\pi$ rd. L'influence de $d$ et $\varepsilon_{\mathrm{p}}$ sur les sections efficaces différentielles se retrouve sur les sections efficaces intégrales (Figs. 6a et 6b)

L'angle de déviation et les sections efficaces différentielles et intégrales, calculés à partir du potentiel de polarisation et utilisés aux faıbles énergıes ioniques, sont déjà abondamment décrits dans la littérature (voir par exemple McDaniel [9])

3.2. TESTS DE VALIDITÉ. - Les sections efficaces différentielles $\sigma\left(\chi, \varepsilon_{\mathrm{r}}\right)$ et intégrales $Q_{\mathrm{m}}\left(\varepsilon_{\mathrm{r}}\right)$ ont été déterminées par une approche classique. L'objectıf de ce paragraphe est de discuter sa validité dans les domaines d'énergies qui nous intéressent (c'est-à-dire jusqu'à quelques dizaines $\mathrm{d}^{\prime} \mathrm{eV}$ ). Les calculs sont effectuées dans le cas du système $\mathrm{Ar}^{+} / \mathrm{Ar}$ où on dispose déjà de quelques mesures de sections efficaces différentielles et qui est choisi notamment en raison de son utilisation dans les plasmas RF de dépôt et de gravure (voir par exemple Surendra $e t$ al. [23]).

Comme les paramètres $\varepsilon_{\mathrm{p}}$ et $d$ du potentiel Lennard-Jones dans le cas du système $\mathrm{Ar}^{+} / \mathrm{Ar}$ ne sont pas donnés dans la littérature, on a procédé à un ajustement de ces paramètres de 


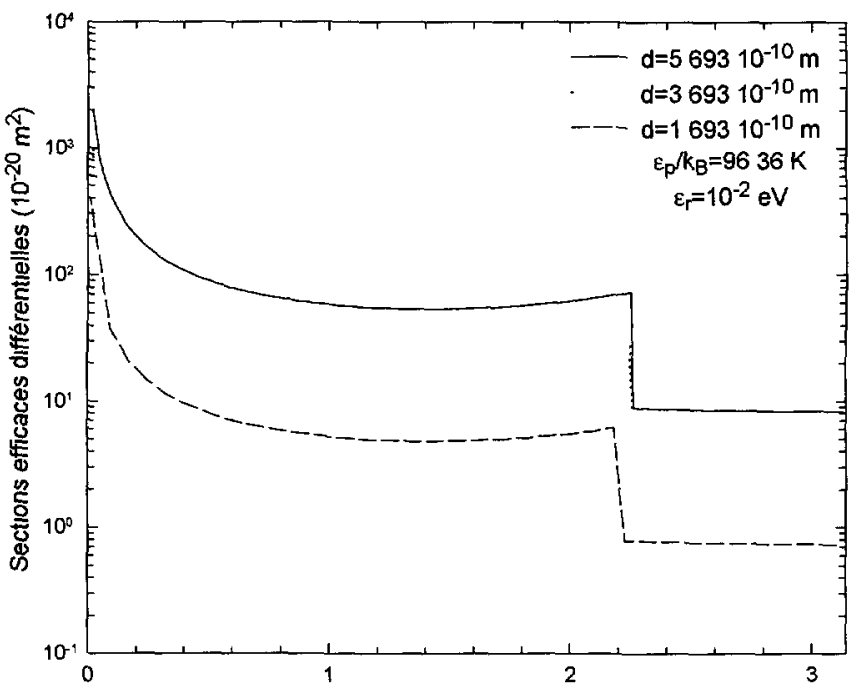

a)

Angle de déviatıon (rd)

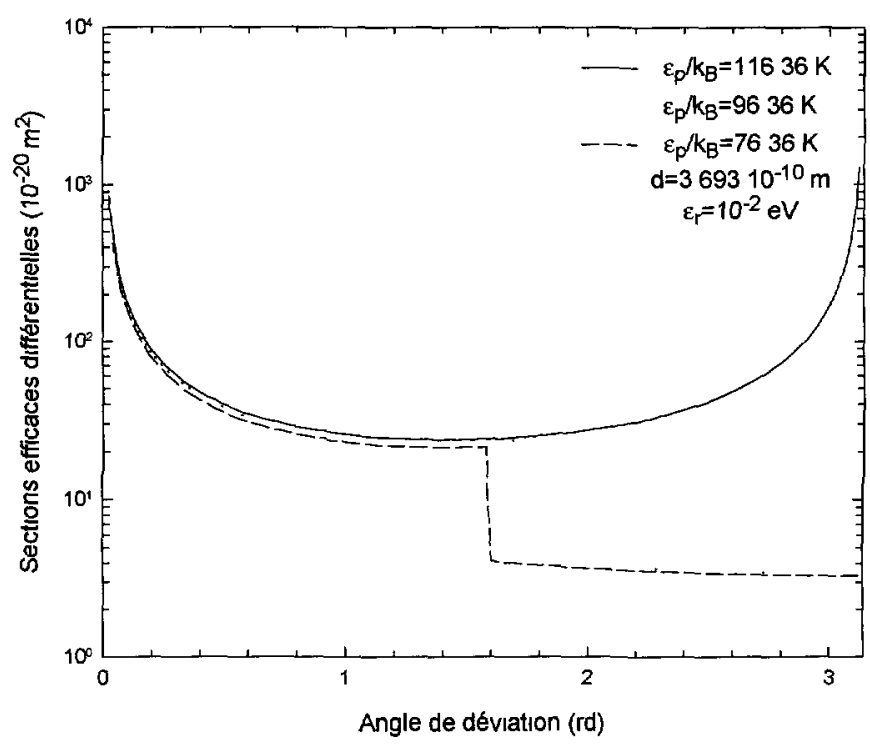

Fig. 5. - a) Section efficace différentielle en fonction de l'angle de déviation pour différentes valeurs du diamètre $d$ de la collision dans le cas d'un potentiel de type Lennard-Jones. b) Section efficace différentielle en fonction de l'angle de déviation pour différentes valeurs de l'énergie du puits $\varepsilon_{\mathrm{p}}$ dans le cas d'un potentiel de type Lennard-Jones.

(a) Differential cross section as a function of the deviation angle for different values of the collision diameter $d$ in the case of the Lennard-Jones potential b) Differential cross section as a function of the deviation angle for different values of the energy of the potential well depth $\varepsilon_{\mathrm{p}}$ in the case of the Lennard-Jones potential.] 


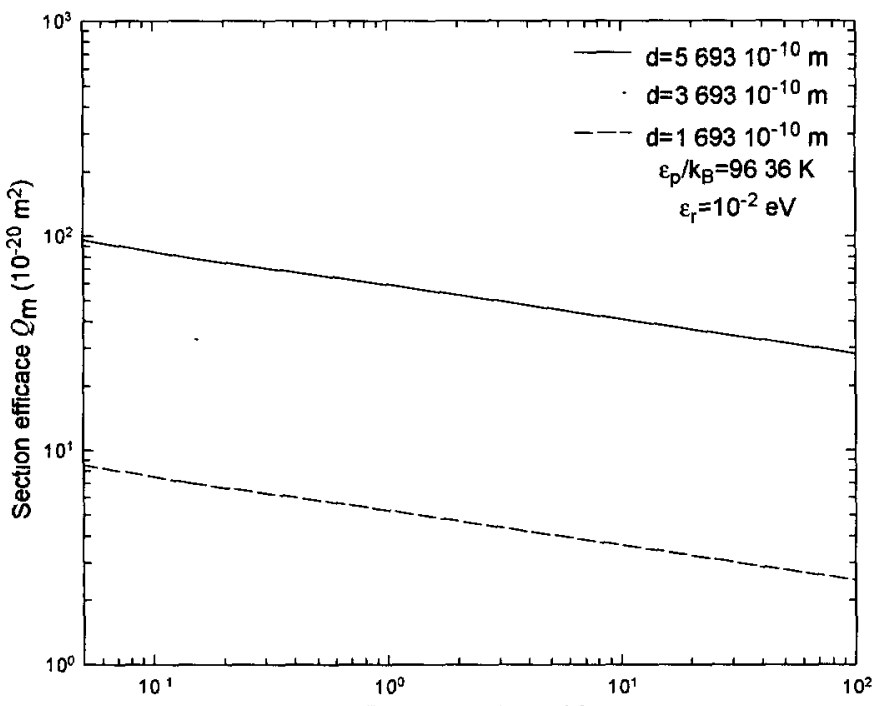

a)

Energie relative (eV)

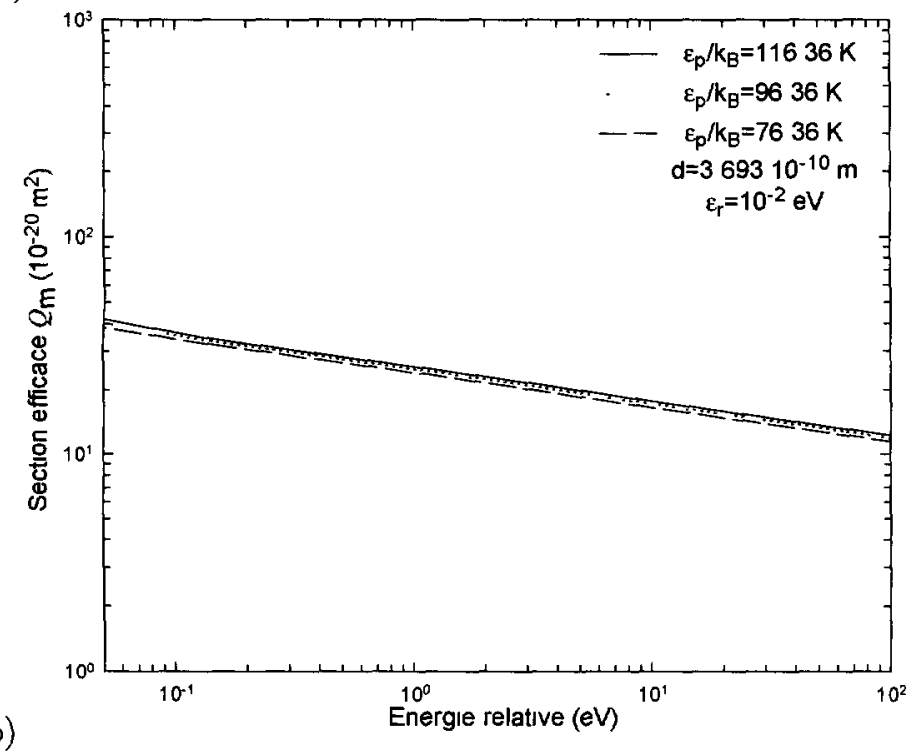

Fig. 6. - a) Section de transfert de la quantité de mouvement en fonction de l 'énergie relative pour différentes valeurs du diamètre de la collision $d$ dans le cas d'un potentiel de type Lennard-Jones b) Section de transfert de la quantité de mouvement en fonction de l 'énergie relative pour différentes valeurs de l'énergie du puits $\varepsilon_{\mathrm{p}}$ dans le cas d'un potentiel de type Lennard-Jones.

(a) Momentum transfer collision cross section as a function of the relative energy for different values the collision diameter $d$ in the case of the Lennard-Jones potential. b) Momentum transfer collision cross section as a function of the relative energy for different values of the energy of the potential well depth $\varepsilon_{\mathrm{p}}$ in the case of the Lennard-Jones potential.] 


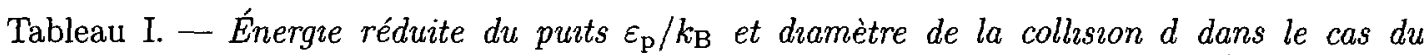
potentiel Lennard-Jones pour le système $\mathrm{Ar} / \mathrm{Ar}$ issus de [1] et pour le système $\mathrm{Ar}^{+} / \mathrm{Ar}$ calculés dans ce travaul.

[Reduced energy of the well depth $\varepsilon_{\mathbf{p}} / k_{\mathrm{B}}$ and collision diameter $d$ in the case of the LennardJones potential for Ar/Ar system from [1] and $\mathrm{Ar}^{+} / \mathrm{Ar}$ system calculated in this work.]

\begin{tabular}{|c|c|c|}
\hline & $\varepsilon_{\mathrm{p}} / k_{\mathrm{B}}(K)$ & $d\left(10^{-10} \mathrm{~m}\right)$ \\
\hline $\mathrm{Ar} / \mathrm{Ar}$ & $124,00[1]$ & $3,418[1]$ \\
\hline $\mathrm{Ar}^{+} / \overline{\mathrm{Ar}}$ & 120,38 & 4,408 \\
\hline
\end{tabular}

telle sorte que les sections efficaces différentielles issues du calcul classique soient en accord avec les mesures de Vestal et al. [24] (voir Figs. 7a et 7b). L'accord observé sur ces figures est assez cohérent avec les mesures y compris aux faibles valeurs de l'angle de déviation car l'incertitude expérimentale est assez importante aux faibles valeurs de $\chi$ (proche de $\pm 60 \%$ ). Il faut souligner que les paramètres $\varepsilon_{\mathrm{p}}$ et $d$ choisis pour commencer les calculs sont ceux correspondant aux interactions neutre-neutre (Ar/Ar). Les paramètres $d$ et $\varepsilon_{\mathrm{p}}$ qui ont donnés le meilleur accord entre mesures et calculs sont représentés dans le Tableau I.

3.3. COEFFICIENTS DE TRANSPORT DANS LE SYSTÈme $\mathrm{Ar}^{+} / \mathrm{Ar}$. - Une fois que les paramètres $d$ et $\varepsilon_{\mathrm{p}}$ du potentiel de Lennard-Jones ont été déterminés et validés à travers les comparaisons effectuées avec les sections efficaces différentielles mesurées pour quelques énergies ioniques, on peut déterminer les sections efficaces différentielles pour l'ensemble de l'intervalle d'énergies nécessaires au code Monte-Carlo sachant qu'en dessous d'environ $0,1 \mathrm{eV}$, les sections efficaces différentielles ont été déterminées à partir du potentiel de polarisation et en dessus à partir du potentiel de Lennard-Jones. La figure $8 \mathrm{a}$ représente la section efficace différentielle ainsi déterminée dans le système $\mathrm{Ar}^{+} / \mathrm{Ar}$ pour des énergies variant jusqu'à $100 \mathrm{eV}$. Le traitement des collisions dans l'algorithme de Monte-Carlo nécessite aussi la connaissance des sections efficaces des processus inélastiques et en particulier celles du transfert de charge résonnant qui est le processus dominant de freinage des ions car il ramène les ions accélérés par le champ électrique sans cesse à l'énergie du gaz qui est dans notre cas un gaz faiblement ionisé à la température ambiante $(300 \mathrm{~K})$. Les sections efficaces des processus élastiques et inélastiques sont représentées sur la figure $8 \mathrm{~b}$ Les coefficients de transport calculés par le code de Monte-Carlo permettant la simulation des expériences classiques de tube de dérive sont représentées sur les figures $9 \mathrm{a}$ et $9 \mathrm{~b}$. On observe un bon accord entre les calculs et les mesures car la barre d'erreur expérimentale est pour les vitesses de dérive et mobilités ioniques (Fig. 9a) de $1 \%$ pour un champ réduit $\mathrm{E} / \mathrm{N} \leq 250 \mathrm{Td}$ et de $3 \%$ pour $\mathrm{E} / \mathrm{N}>250 \mathrm{Td}$ [25]. L'incertitude expérimentale sur l'énergie caractéristique donnée dans la compilation d'Ellis et al. [26] est de l'ordre de $10 \%$. La figure $9 \mathrm{~b}$ représente notamment les énergies caractéristıques transverse $D_{\mathrm{T}} / \mu$, longitudinale $D_{\mathrm{L}} / \mu$ et isotrope $D_{0} / \mu$ calculées par simulation de Monte-Carlo, l'énergie $D_{0} / \mu$ étant définie par : $D_{0} / \mu=\left(2 D_{\mathrm{T}} / \mu+D_{\mathrm{L}} / \mu\right) / 3$. On remarque que les mesures sont en bon accord, non pas avec $D_{\mathrm{T}} / \mu$, mais avec $D_{0} / \mu$ qur est la quantité souvent mesurée dans la littérature par les techniques de tube de dérive (voir McDaniel et Mason [28]). Ces accords cohérents avec les incertitudes expérimentales sont dus au bon choix aussi bien des sections efficaces différentielles qui ont été calculées à partir des potentıels d'interaction que des sections efficaces intégrales et de transfert de charge résonnant. 


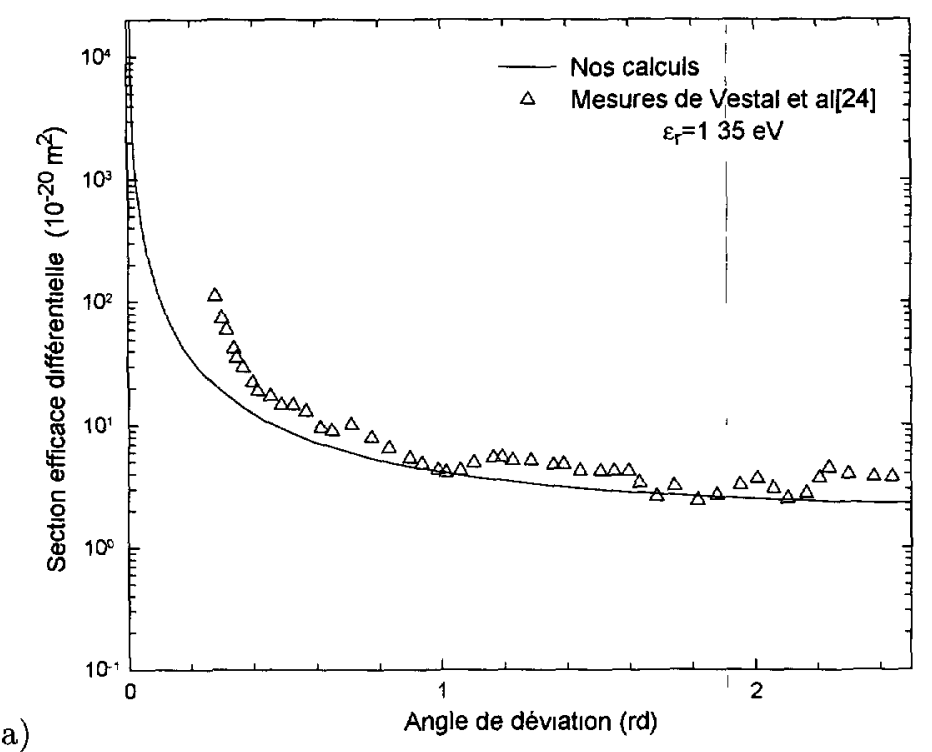

a)

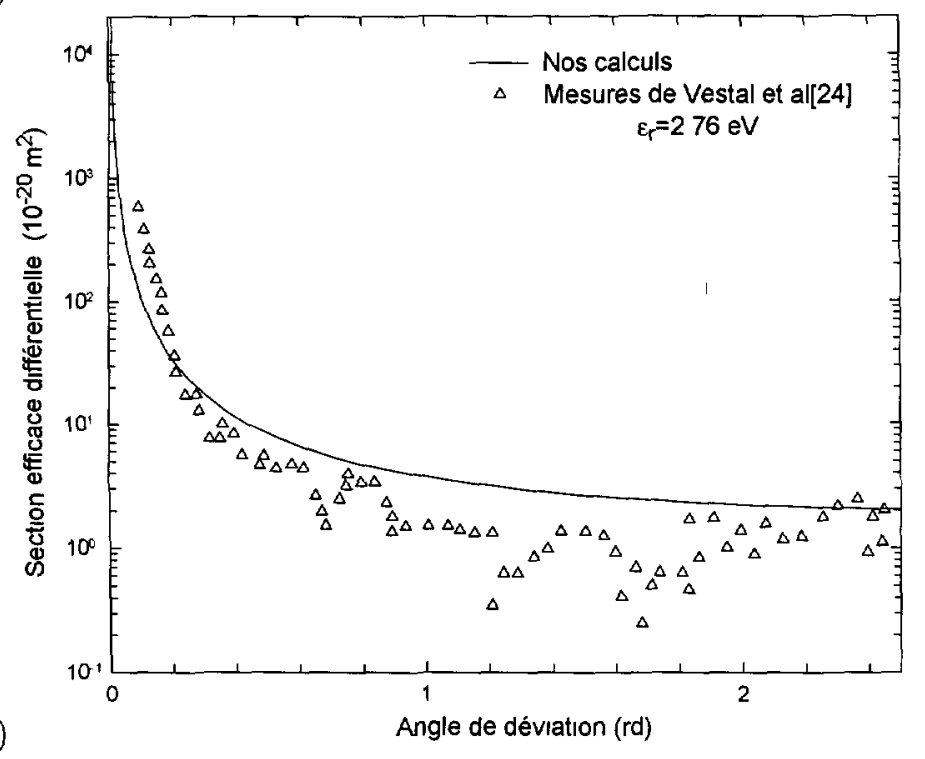

Fig. 7. - a) Section efficace différentielle calculée à partır du potentiel Lennard-Jones $[6,12]$ et mesurée par Vestal et al. [24] pour une énergie relatıve $\varepsilon_{\mathrm{r}}=1,35 \mathrm{eV}$. b) Section efficace différentielle calculée à partir du potentiel Lennard-Jones $[6,12]$ et mesurée par Vestal et al. [24] pour une énergie relative $\varepsilon_{\mathrm{r}}=2,76 \mathrm{eV}$.

[a) Differential cross section calculated from the Lennard-Jones [6,12] potential and measured by Vestal et al. [24] for the relative energy $\varepsilon_{\mathrm{r}}=1.35 \mathrm{eV}$. b) Differential cross section calculated from the Lennard-Jones [6,12] potential and measured by Vestal et al. [24] for the relative energy $\varepsilon_{\mathfrak{r}}=2.76 \mathrm{eV}$.] 
a)
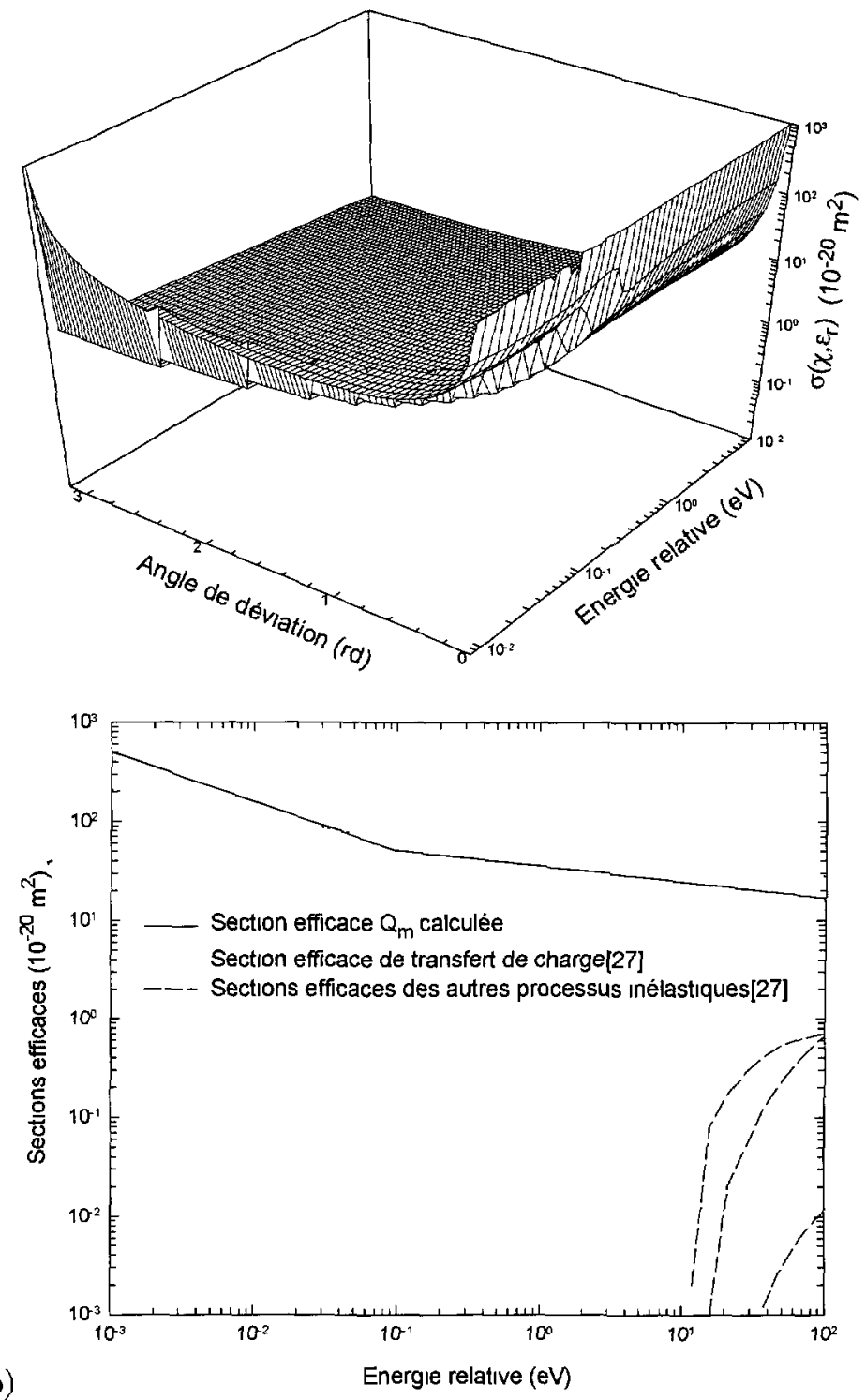

Fıg. 8 - a) Sectıon efficace différentielle de collisıon élastıque en fonction de l'angle de déviation et de l'énergie relatıve dans le cas du système $\mathrm{Ar}^{+} / \mathrm{Ar}$. b) Jeu de sections efficaces intégrales élastique et inélastıques en fonction de l'énergie relative dans le cas du système $\mathrm{Ar}^{+} / \mathrm{Ar}$

(a) Differential cross section as a function of the deviation angle and the relative energy in the case of the system $\mathrm{Ar}^{+} / \mathrm{Ar}$ b) Set of the integral elastic and inelastic collision cross sections as a function of the relative energy in the case of the system $\mathrm{Ar}^{+} / \mathrm{Ar}$.]

\section{Conclusion}

Cet article a été consacré à la détermination des sections efficaces différentielles et intégrales ion- atome nécessaires au calcul des coefficients de transport des ions dans les gaz faiblement 


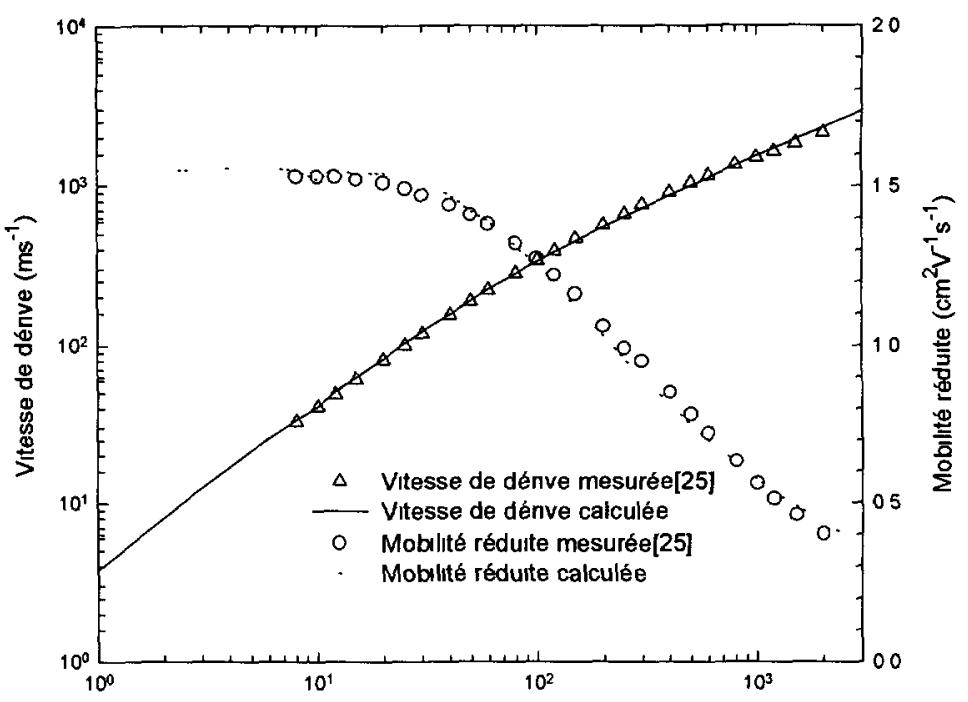

a)

$E / N(T d)$

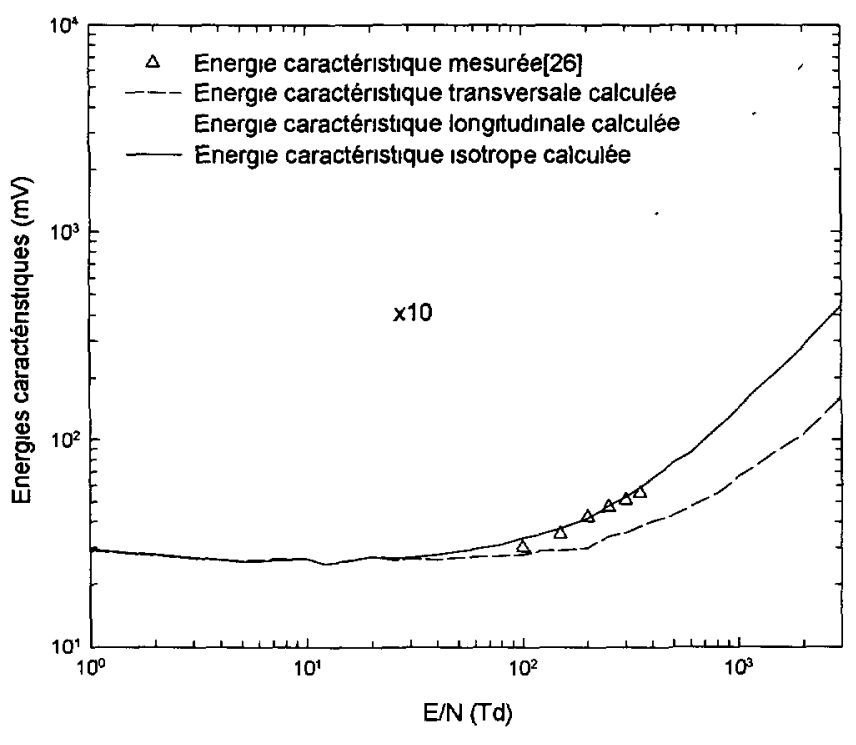

Fig. 9. - a) Vitesse de dérive et mobilité en fonctıon du champ réduit $\mathrm{E} / \mathrm{N}$ dans le cas des ions $\mathrm{Ar}^{+}$ dans Ar mesurées [25] et calculées dans ce travall b) Énergies caractéristiques en fonction du champ réduit $\mathrm{E} / \mathrm{N}$ dans le cas des ions $\mathrm{Ar}^{+}$dans $\mathrm{Ar}$.

(a) Drift velocity and mobility as a function of the reduced field $\mathrm{E} / \mathrm{N}$ in the case of $\mathrm{Ar}^{+}$ions in $\mathrm{Ar}$ gas measured [25] and calculated in the present work b) Characteristic energies as a function of the reduced field $\mathrm{E} / \mathrm{N}$ in the case of $\mathrm{Ar}^{+}$lons in $\mathrm{Ar}$ gas.]

ionisés. Dans le cas du système $\mathrm{Ar}^{+} / \mathrm{Ar}$ et pour des intervalles d'énergie allant jusqu'à quelques dizaines d'eV, les sections efficaces ont été obtenues à partir des potentiels d'interaction de polarisation pour les faibles énergies (en dessous de 0,1 eV) et de Lennard-Jones pour les énergies plus élevées. La méthode de calcul des sections efficaces basée sur la mécanique classique a 
d'abord été validée par comparaison des sections efficaces différentielles mesurées et calculées. Les paramètres du potentiel Lennard-Jones ont ainsi été déterminés pour la première fois dans la littérature dans le cas du système $\mathrm{Ar}^{+} / \mathrm{Ar}$. Ensuite, ces sections efficaces ont été utilisées dans un code de simulation statistique de Monte-Carlo du transport des ions $\mathrm{Ar}^{+}$dans l'Argon à la température ambiante $(300 \mathrm{~K})$. Les coefficients de transport (vitesse de dérive, mobilité ionique et coefficient de diffusion) obtenus sont en bon accord avec les mesures de tube de dérive de la littérature confirmant ainsi la validation de la méthode de détermination des sections efficaces.

Dans le futur, les mêmes méthodes de détermination des sections efficaces différentielles permettant de prendre en compte l'effet de l'anisotropie des collisions seront appliquées au cas des ions atomiques et moléculaires $\left(\mathrm{O}^{+}, \mathrm{O}_{2}^{+}, \mathrm{N}^{+}, \mathrm{N}_{2}^{+}\right.$, etc. $)$dans les gaz moléculaires $\left(\mathrm{N}_{2}, \mathrm{O}_{2}\right)$ et leurs mélanges.

\section{Bibliographie}

[1] Hirschfelder J.O., Curtiss C.F., and Bird R.B., Molecular Theory of Gases and Liquids (J. Wiley, New York, 1964) pp. 21.

[2] Hasted J.B. and Phil M.A.D., Physics of Atomic Collisions (Second Edition, Ed., Butterworths, London, 1972) pp. 57.

[3] Delcroix J.-L. et Bers A., Physique des Plasmas (InterEditions, CNRS Editions, Tome 1, Paris, 1994) pp. 143.

[4] Hirschfelder J.O., and Meath W.J., The nature of intermolecular forces, Adv. Chem. Phys. 12 (1967) 3-106.

[5] Certain P.R. and Bruch L. W., Intermolecular forces, in MTP International Review of Science, Physical Chemistry Series One, Vol. 1, Theoretical Chemistry, W. Byers-Brown (Ed., Butterworth, London, 1972) Chap. 4.

[6] Maitland G.C., Rigby M., Smith E.B., and Wakeham R., Intermolecular Forces. Their Origin and Determination (Oxford University Press, Oxford, 1981).

[7] Jancel R. et Kahan Th., Electrodynamique des Plasmas (Dunod, Paris, 1963).

[8] Gray C.G. and Gubbins K.E., Theory of Molecular Fluids (Oxford University Press, Oxford, 1984) Vol. 1.

[9] McDaniel E.W., Collision Phenomena in Ionized Gases (J. Willey, New York, 1964) Chap. 3.

[10] Lin S.L. and Bardsley, Monte-Carlo simulation of ion motion in drift tubes, J. Chem Phys. 66 (1977) 435.

[11] Dickinson A. S. and Ruchards D., Classical small-angle anisotropic potential scattering, $J$. Phys. B: Atom. Molec. Phys. 12 (1979) 3005.

[12] Lübbert A. and Rotzoll G., Monte-Carlo averaging technique for analysis of elastic laboratory differential cross section data J. Phys. E: Scl. Instrum. 11 (1978) 63.

[13] Cupini E. and De Matteis A . Plasma-Neutral elastic collisions in Monte-Carlo computational models, $R$ Nuovo Cimento 11D (1989) 1489.

[14] McDaniel E. W., Mitchell J. B A., and Rudd M. E., Atomic Collisions Heavy Particle Projectiles (J. Wiley, New York, 1993) Chap. 1.

[15] McDowell M.R.C. and Coleman J.P., Introduction to the Theory of Ion-Atom Collisions (North-Holland Publishing Company, Amsterdam, 1970) Chap 1. 
[16] Pauly H. and Toennies J.P., The Study intermolecular Potentials with Molecular Beams at Thermal Energies (Advances in Atomic and Molecular Physics, Ed., Academic Press Inc., New York, Vol. 1. 1965) pp. 195.

[17] Bransden B H. and Joachain C.J., Physics of Atoms and Molecules (J. Wiley, New York, 1983).

[18] Mason E. A., Scattering of low velocity molecular beams in gases, J. Chem. Phys. 26 (1957) 667

[19] Hennad A., Kinetics of ions in molecular gases from classical and optımized Monte-Carlo simulations. Determination of basic data in arr, Thèse de Doctorat d'Université Paul Sabatier de Toulouse, N 2458 (1996).

[20] Yousfi M., Hennad A., and Alkaa A., Monte-Carlo simulation of electron swarms at low reduced electrıc fields, Phys. Rev. E 49 (1994) 3264.

[21] Hennad A. and Yousfi M., Anisotropy collision effect on ion transport in cold gas discharges with Monte-Carlo simulation (International Conference on Phenomena in Ionized Gases ICPIG XXII, Stevens Institute of Technology, Hoboken. New Jersey, USA, July 31stAugust 4th) 2 (1995) 53

[22] Johnson R. E., Introduction to Atomic and Molecular Collisions (Plenum Press, New York, 1982) Chap. 1.

[23] Surendra M., Graves D.B. and Jellum G.M , Self-consistent model of a direct-current glow discharge: treatment of fast electrons, Phys Rev A 41 (1990) 1112-1125

[24] Vestal M.L., Blakley C.R., and Futrell J.H., Crossed-beam measurements of differentral cross sections for elastic scattering and charge exchange in low-energy $\mathrm{Ar}^{+}-\mathrm{Ar}$ collisions, Phys. Rev. A 17 (1978) 1337.

[25] Ellis H.W., Pai R.Y., McDaniel E W., Mason E.A., and Viehland L.A., Transport properties of gaseous ions over a wide energy range, Atom. Data and Nucl. Data Tables 17 (1976) $177-210$.

[26] Ellis H.W, Thackston M.G., McDaniel E.W., and Mason E.A., Transport properties of gaseous ions over a wide energy range. Part III, Atom. Data and Nucl. Data Tables 31 (1984) 113-151

[27] Phelps A.V., Cross sections and swarm coefficients for Nitrogen ions and Neutrals in N2 and Argon ions and Neutrals in Ar for energies from $0.1 \mathrm{eV}$ to $10 \mathrm{keV}, J$. Phys. Chem. Ref. Data 20 (1991) 557-573.

[28] McDaniel E.W., Mason E.A., The mobility and diffusion of ions in gases ( $\mathrm{J}$ Willey, New York, 1974) 